\title{
Integration of differential ion mobility on a Q-Exactive hybrid quadrupole Orbitrap mass spectrometer
}

Sibylle Pfammatter ${ }^{1,2 \#}$; Zhaoguan $\mathrm{Wu}^{1,2 \#}$; Eric Bonneil ${ }^{1}$; Derek J. Bailey ${ }^{3}$; Satendra Prasad $^{3}$; Michael Belford ${ }^{3}$; Jonathan Rochon ${ }^{4}$; Pierre Picard ${ }^{4}$; Jean Lacoursière ${ }^{4}$; Jean-Jacques Dunyach ${ }^{3}$; Pierre Thibault ${ }^{1,2}$

${ }^{1}$ Institute for Research in Immunology and Cancer (IRIC); ${ }^{2}$ Department of Chemistry, Université de Montréal, Montréal, QC, ${ }^{3}$ ThermoFisher Scientific, San Jose, CA, ${ }^{4}$ Phytronix Technologies, Québec, QC

"co-first authors

Correspondence : Pierre Thibault, Phone: (514)-343-6910. Fax: (514) 343-7586. E-mail: pierre.thibault@umontreal.ca 
Table of contents:

Supplementary material and methods.

Supplementary Figure 1: Mounting flange to interface the FAIMS Pro ${ }^{\mathrm{TM}}$ on the Q-Exactive mass spectrometer.

Supplementary Figure 2: Distribution of BSA tryptic peptides according the CV..

Supplementary Figure 3: Distribution and frequency of tryptic peptides identified from HeLa cells in LC-MS/MS experiments with and without FAIMS.

Supplementary Figure 4: Identification of HeLa tryptic peptides by LC-MS-MS using SIFT and FAIMS.

Supplementary Figure 5: Impact of interference on quantitative measurements.

Supplementary Figure 6: Scatter plots of interference free index (a) and fold changes (b) obtained with and without FAIMS and SIFT.

Supplementary Figure 7: Boxplot distribution of fold changes for peptides grouped by IFI.

Supplementary Table 1: Scanning ranges used for full MS scan and SIFT with and without FAIMS - Included in this document

Supplementary Table 2 (.xls): LC-MS/MS analyses of HeLa digest with and without FAIMS (separate file)

Supplementary Table 3 (.xls): LC-MS/MS analyses of HeLa digest with and without FAIMS using mz segments (SIFT) (separate file)

Supplementary Table 4 (.xls): LC-MS/MS analyses of HeLa digest with and without FAIMS using $\mathrm{mz}$ segment stepping. (separate file)

Supplementary Table 5 (.xls): LC-MS/MS analyses of two proteome model with and without FAIMS using $\mathrm{m} / \mathrm{z}$ segment stepping. (separate file) 


\section{Supplementary Material and Methods}

Direct infusion. Bovine Serum Albumin (BioShop, ALB00150) was resuspended in 50 $\mathrm{mM}$ ammonium bicarbonate (Sigma Aldrich, A6141), reduced with 5mM TCEP (Thermo Fisher Scientific, 77720) and alkylated with 10mM 2-chloroacetamide (Sigma Aldrich, C0267) prior to overnight incubation with trypsin (Promega, V511A, enzyme/protein ratio $1: 50$ ) at $37^{\circ} \mathrm{C}$. The protein digest was desalted on an Oasis HLB extraction cartridge (Waters, WAT094225). For direct infusion, peptides were diluted to $0.4 \mu \mathrm{g} / \mu \mathrm{L}$ in $50 \%$ methanol (Fisher Scientific, A4524) $/ 0.2 \%$ formic acid (FA) (Fisher Scientific, AC147930010).

Protein extraction and enzymatic digestion. For LC-MS benchmark tests, we have injected 500ng of Thermo Scientific ${ }^{\mathrm{TM}}$ Pierce ${ }^{\mathrm{TM}} \mathrm{HeLa}$ protein digest standard (Thermo Fisher Scientific, 88329) diluted in $0.2 \%$ aqueous FA. For tandem mass tag (TMT) experiments, Saccharomyces cerevisiae and human embryonic kidney 293 (HEK293) cells were twice washed with cold Phosphate Buffered Saline (PBS) (Fisher Scientific, BP399-1) and pelleted by centrifugation (1000 rpm, 5min). Lysis buffer containing 8M Urea (Fisher Scientific, BP169), 50mM HEPES (Bio Basic Inc., HB0264) and 75mM sodium chloride (Fisher Scientific, BP358), $\mathrm{pH} 8.2$ was added to the cell pellets. Yeast cells were mechanically lysed with $10 \mathrm{~min}$ bead beating, HEK293 cells were mechanically lysed with 2x 10s sonication bursts. Lysates were centrifuged at $14,000 \mathrm{~g}$ for $10 \mathrm{~min}$, and protein concentration of clear lysate was determined by Bradford assay. Proteins were precipitated using methanol/chloroform (lysate/methanol/chloroform/water 1:4:1:3 volume parts). Samples were vortexed and centrifuged for $5 \mathrm{~min}$ at $14,000 \mathrm{~g}$. The precipitated protein layer was washed twice with four volume parts of methanol and air-dried. For tryptic digestion, proteins were dissolved in 200mM HEPES, $\mathrm{pH} 8.2$ and reduced, alkylated and trypsinized (enzyme/protein ratio 1:50) overnight at $37^{\circ} \mathrm{C}$. 

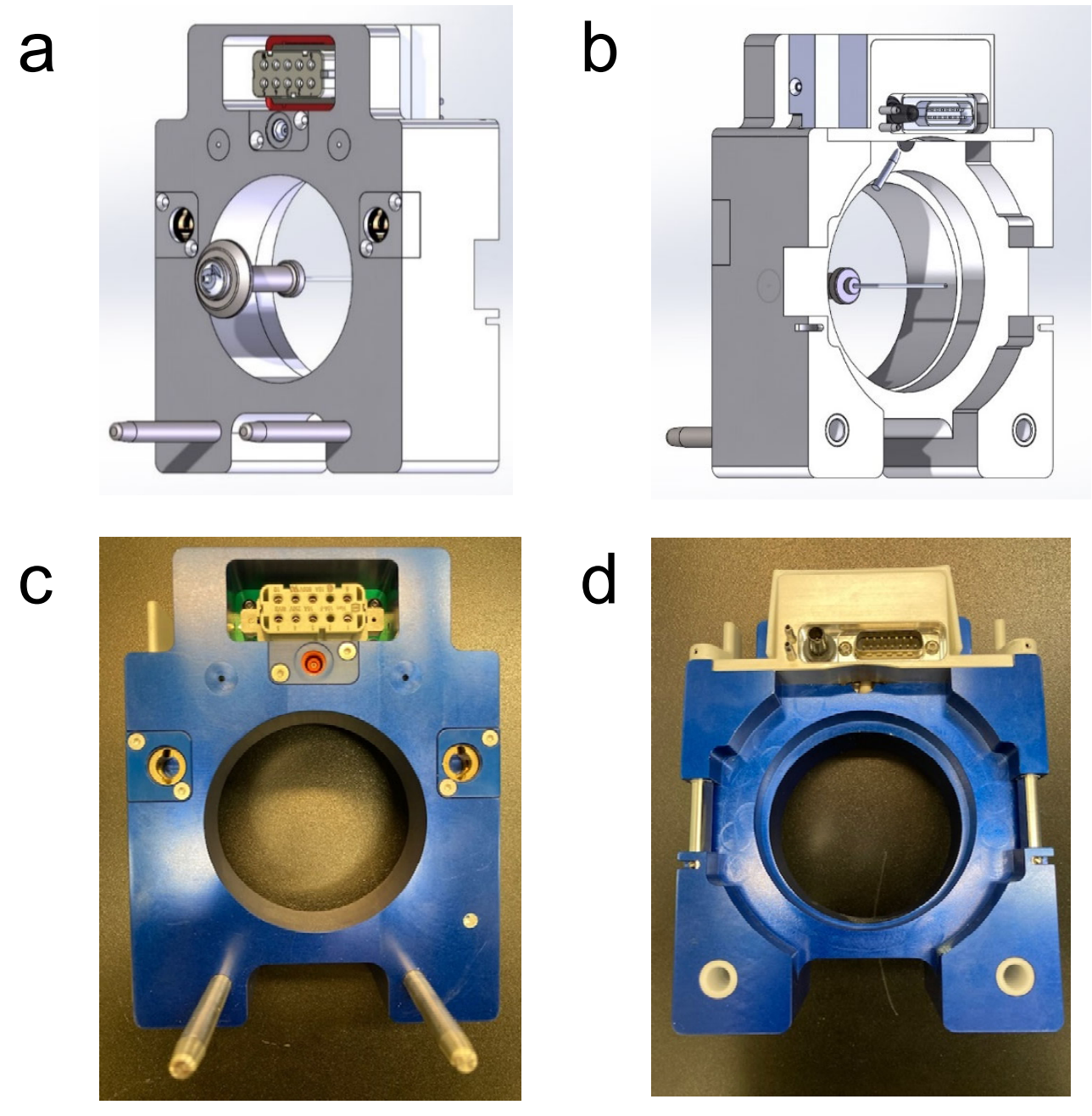

Supplementary Figure 1: Mounting flange to interface the FAIMS Pro $^{\mathrm{TM}}$ on the Q-Exactive mass spectrometer. Schematic representation of the front (a) and back (b) of the mounting flange along with photographs of the front (c) and back (d) of corresponding adapter plate. The flange was designed to mount the FAIMS Pro ${ }^{\mathrm{TM}}$ with an IonMax New Generation interface connector to a Q-Exactive mass spectrometer with an IonMax Old Generation interface. The flange is $170.5 \mathrm{~mm}$ high by $133 \mathrm{~mm}$ wide. The Ion Transfer Tube normally installed on the QExactive has a length of 58.42mm (P / N 70005-20606) and an internal diameter of $0.58 \mathrm{~mm}$. The new Ion transfer Tube has a length of $101.6 \mathrm{~mm}(\mathrm{P} / \mathrm{N}$ 97055-20199). In order to make the electronic and gas interconnections, and the mechanical connectors for the two types of interfaces, the flange has a thickness of $52.29 \mathrm{~mm}$ and correspond to the difference between the length of the two Ion transfer tubes. We therefore used a $101.6 \mathrm{~mm}$ Ion Transfer Tube available from Thermo (P / N 97055-20199). An extension was made to be able to install the $101.6 \mathrm{~mm}$ Ion Transfer Tube on the inlet port of the QExactive.

For electronic interconnections a standard Dsub 15 male connector was used on the IonMax Old Generation inlet side while a female Harting connector (Han 10A-BU-C, P / N: 09200103101) was used on the IonMax New side (FAIMS Pro ${ }^{\mathrm{TM}}$ ). To make the high voltage interconnection, a male panel mount threaded connector from Connectronics Corp. was used. (P / N 10334-02) on the IonMax Old Generation side and a "Straight SCID" connector from Connectronics Corp (P / N 10130-02) on the IonMax New Generation side. 

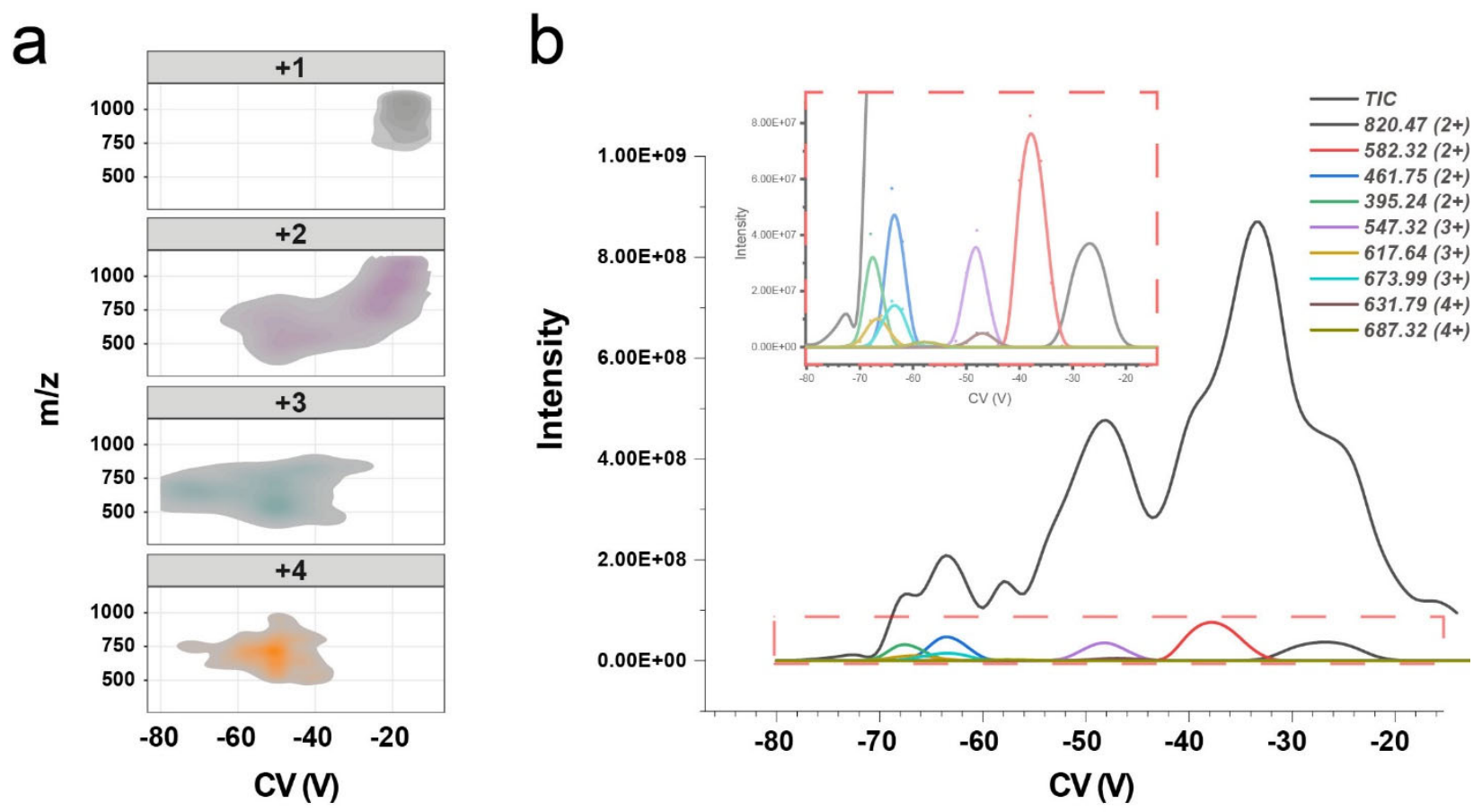

Supplementary Figure 2: Distribution of BSA tryptic peptides according the CV. Direct infusion of BSA tryptic digest by ESI-FAIMS-MS by switching the CV in 2-V steps from $-10 \mathrm{~V}$ up to $-80 \mathrm{~V}$ at DV -4800 . (a) Heat map showing transmission domain for each charge state. (b) Total ion chromatogram (black) with representative extracted ion chromatograms of selected BSA tryptic peptides. 

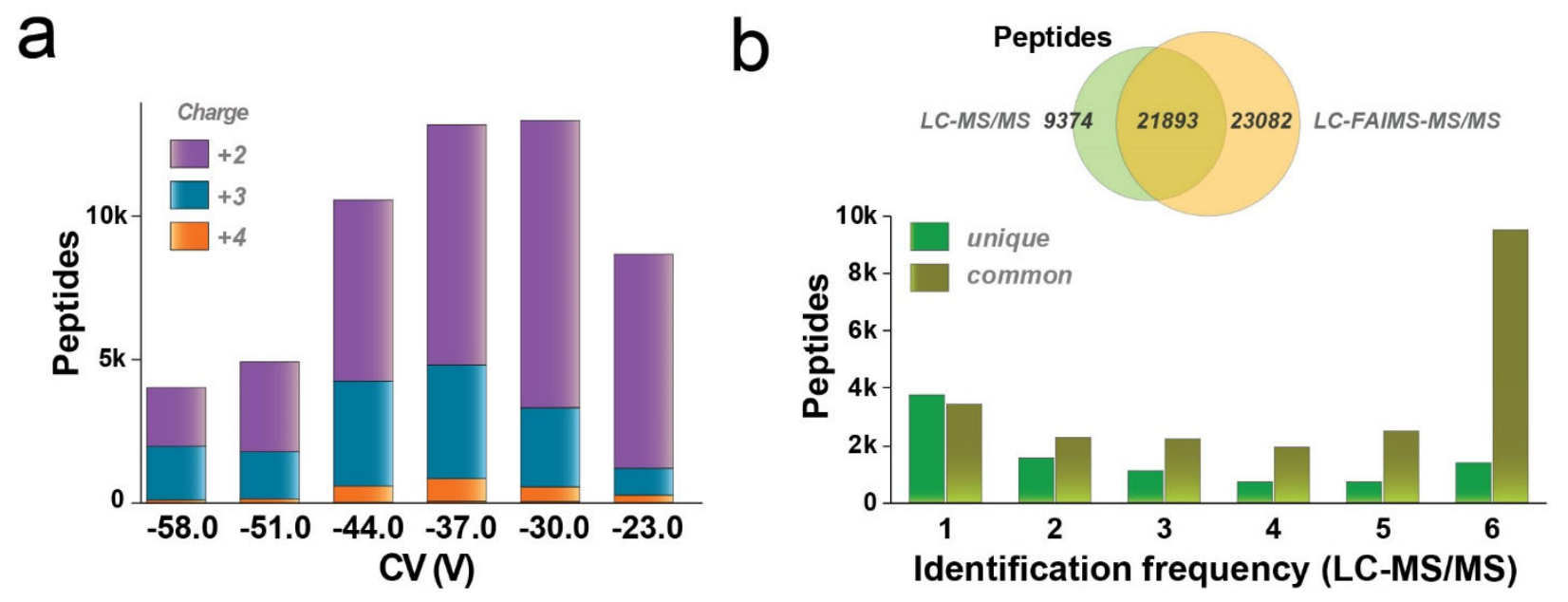

Supplementary Figure 3: Distribution and frequency of tryptic peptides from HeLa cells identified in LC-MS/MS experiments with and without FAIMS. (a) Charge state distribution of HeLa peptides identified with FAIMS. (b) Identification frequency of peptides identified by LC-MS/MS only or identified by LC-MS/MS and LC-FAIMS-MS/MS, 
a

CV (V)

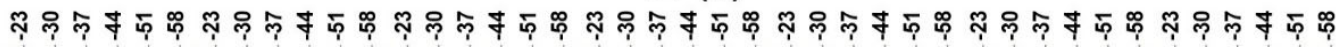
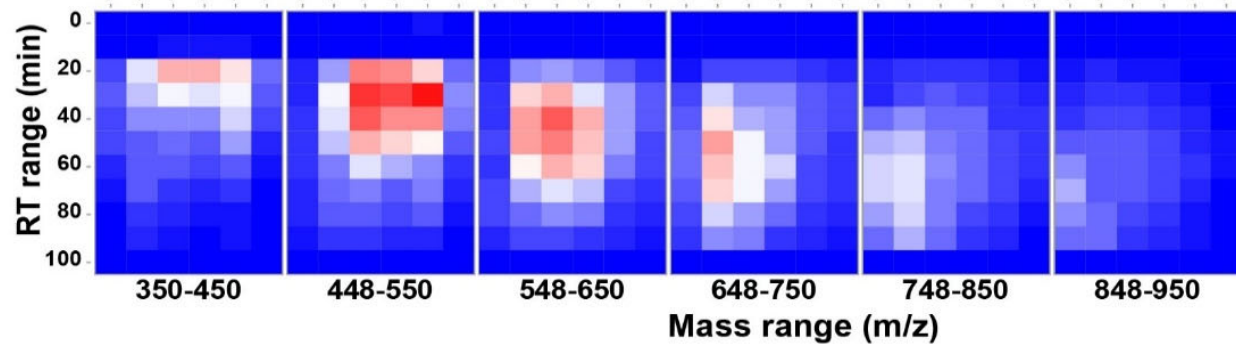

848-950

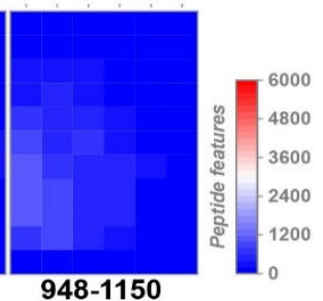

b

CV (V)

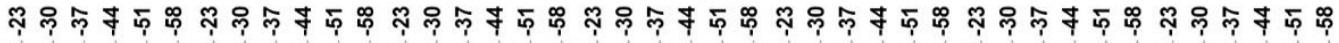
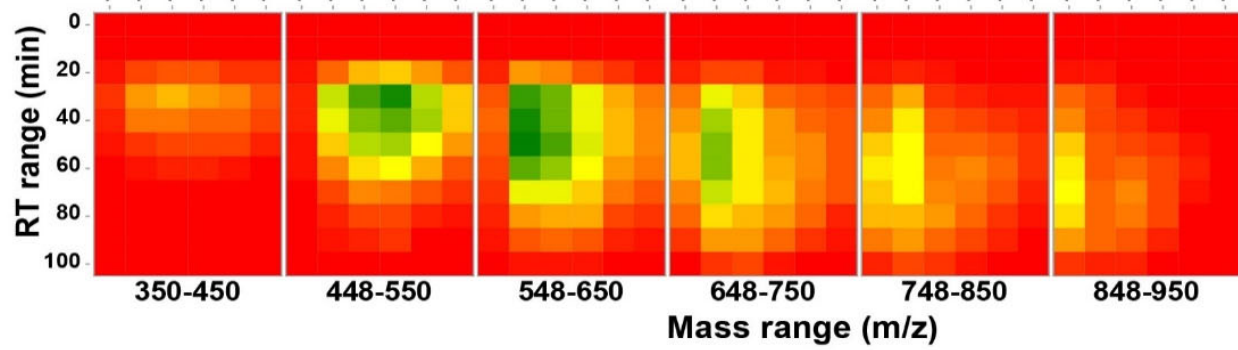

848-950

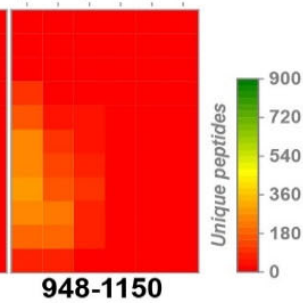

C

m/z segment switching

$350 \quad 400 \quad 450 \quad 500 \quad 550 \quad 600 \quad 650 \quad 700 \quad 750 \quad 800 \quad 850 \quad 900 \quad 950 \quad 1000 \quad 1050 \quad 1100 \quad 1150$

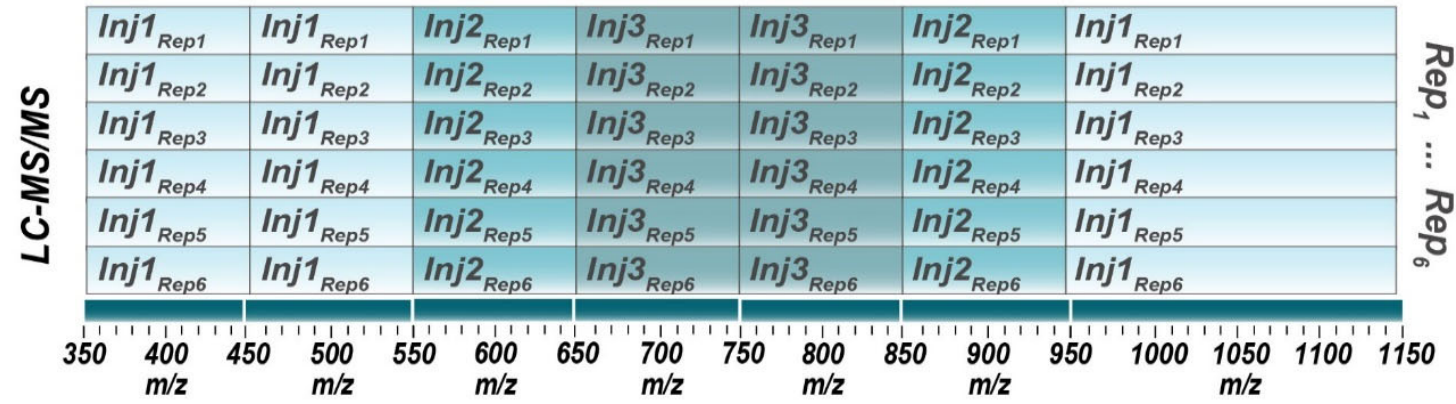

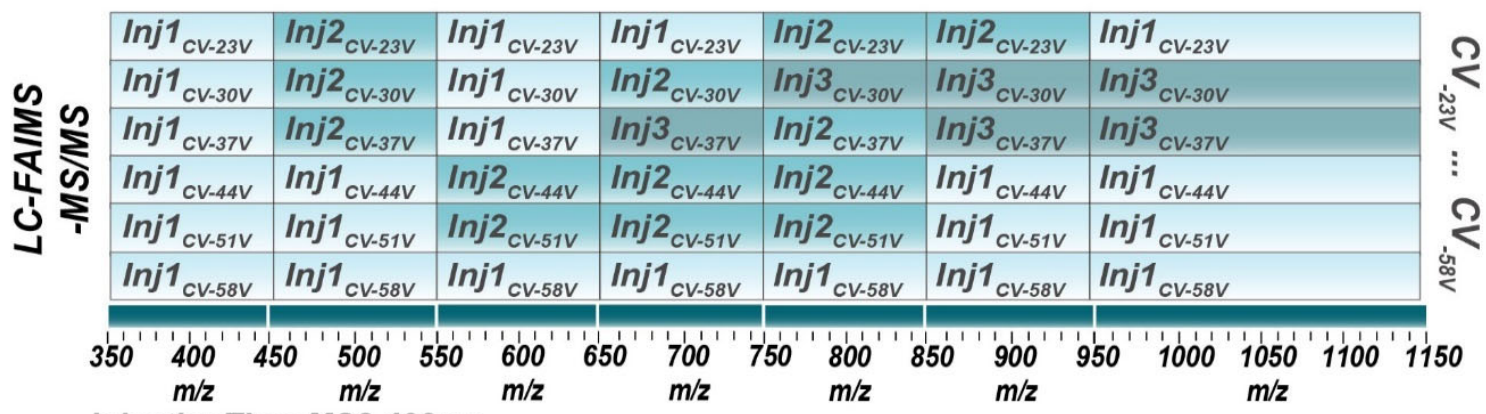

InjectionTime MS2 400ms

Supplementary Figure 4: Identification of HeLa tryptic peptides by LC-MS-MS using SIFT and FAIMS. Heatmap of detected features (a) and unique peptides (b) according to $\mathrm{CV}, \mathrm{m} / \mathrm{z}$ tiles and retention time bins. (b) Schematic representation of the $\mathrm{m} / \mathrm{z}$ cycling per replicate injections with and without FAIMS. 
a

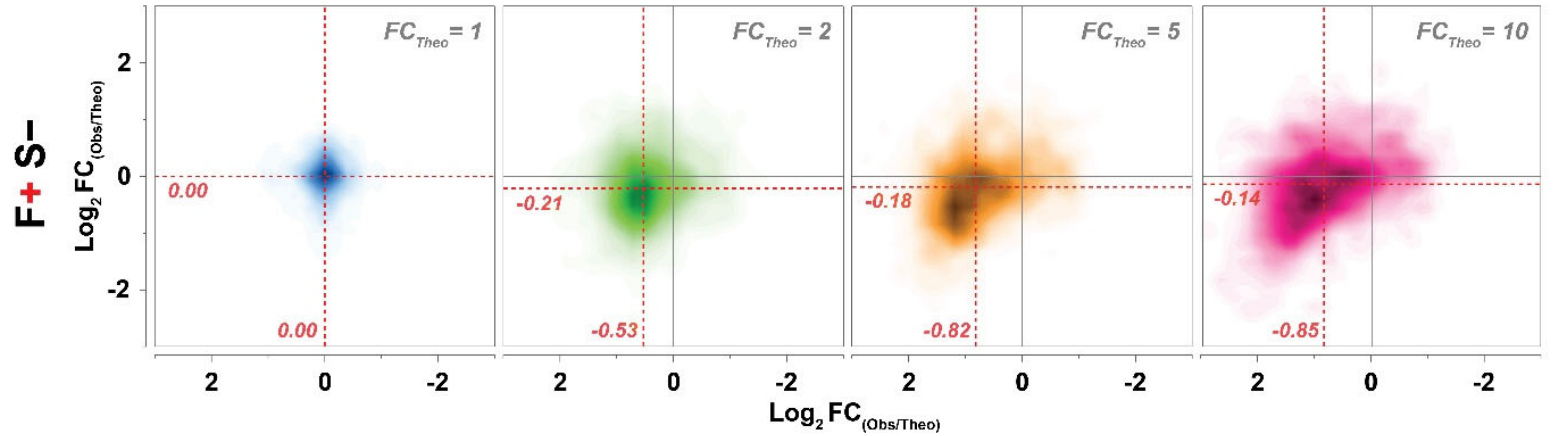

F-S-

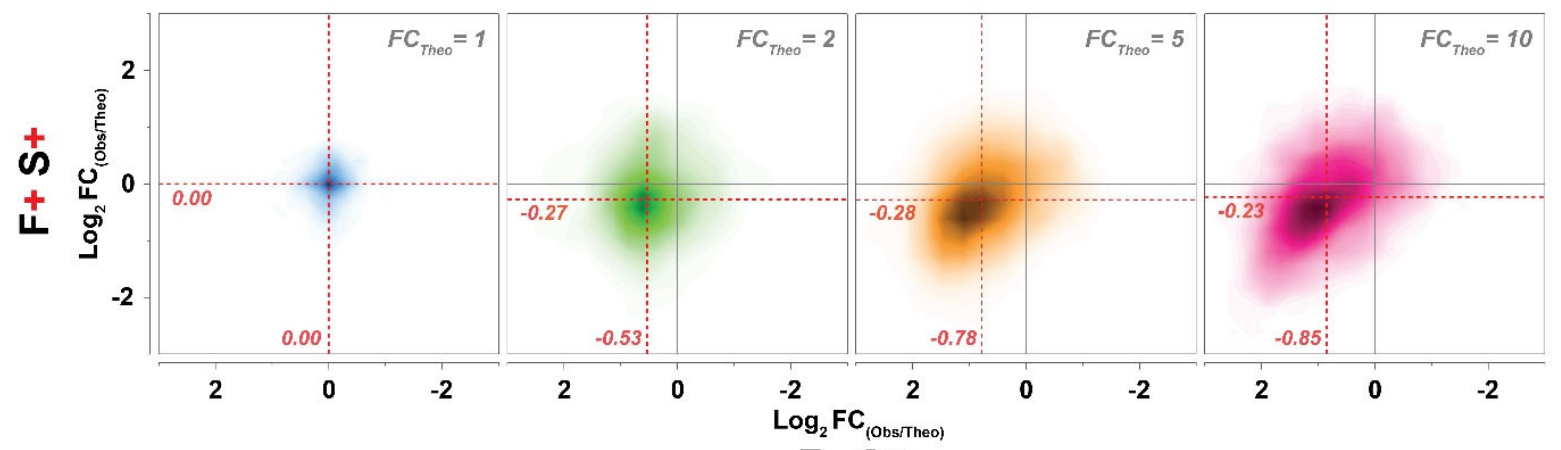

b

F- S+
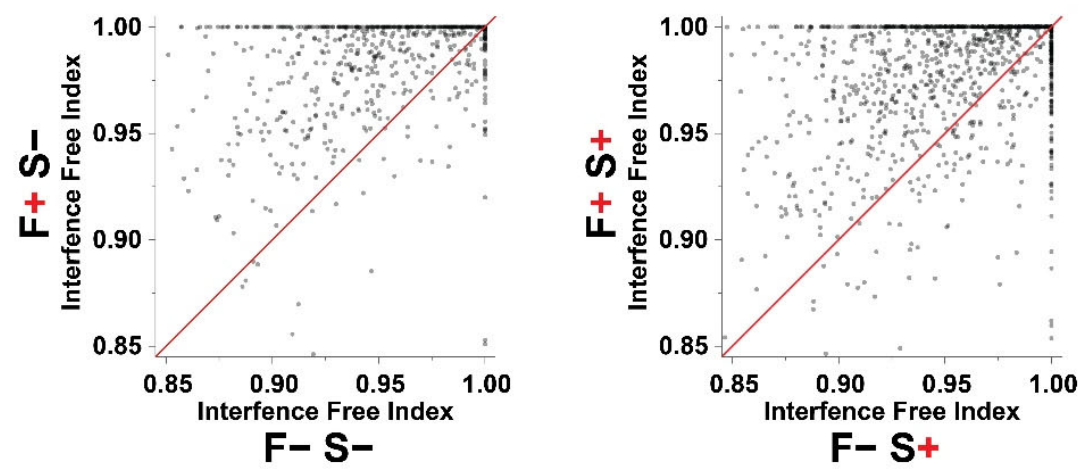

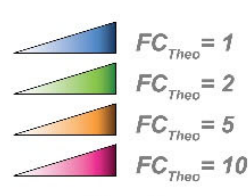

Density

F FAIMS

S Segments

$-/+$ Without / With

Supplementary Figure 5: Impact of interference on quantitative measurements. (a) Deviation of observed from expected TMT ratios with and without FAIMS. (b) Scatter plot of interference free index for common peptides identified with and without SIFT and FAIMS. 

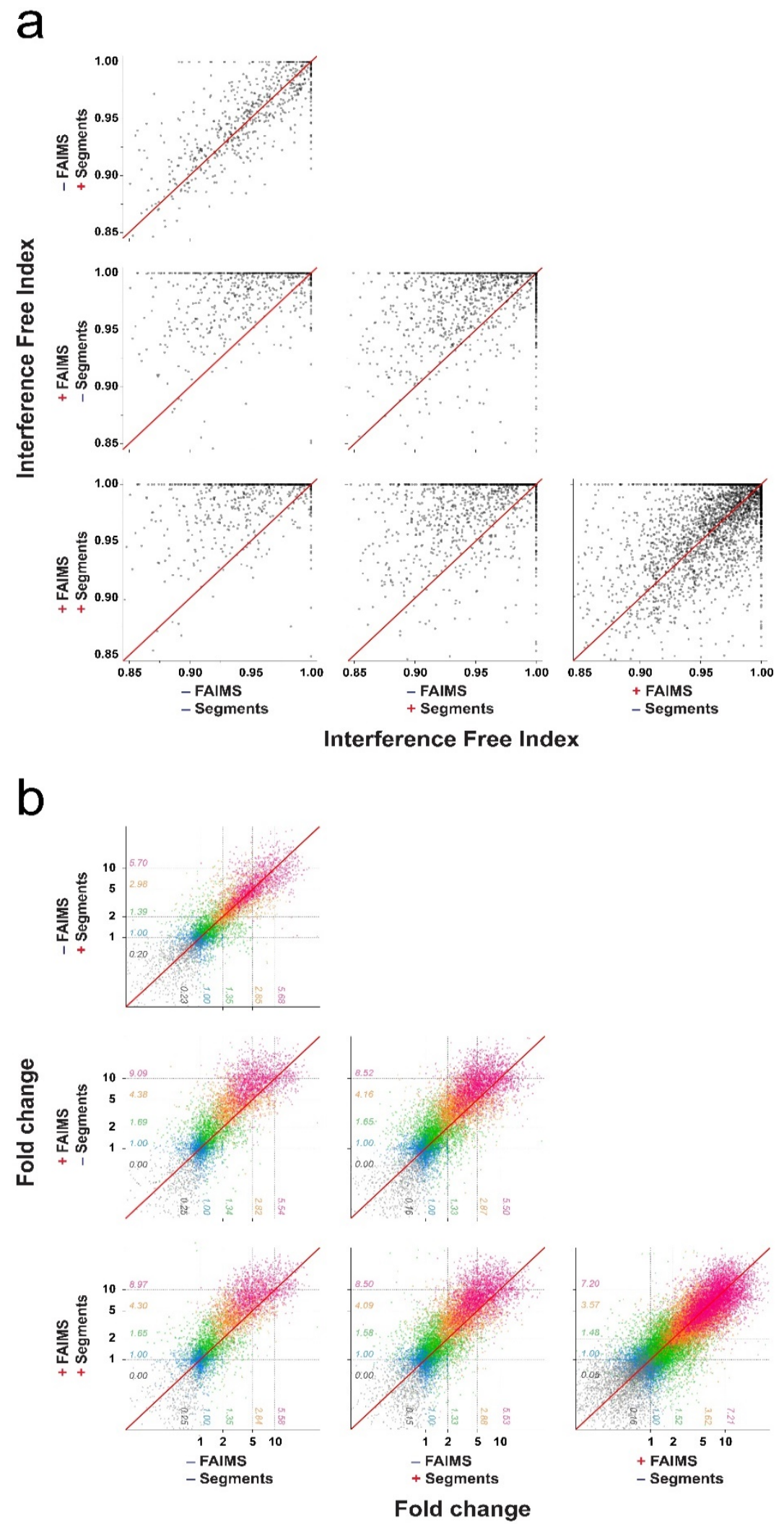

Supplementary Figure 6: Scatter plots of a) interference free index and b) fold changes obtained with and without FAIMS and SIFT. 
Supporting Information

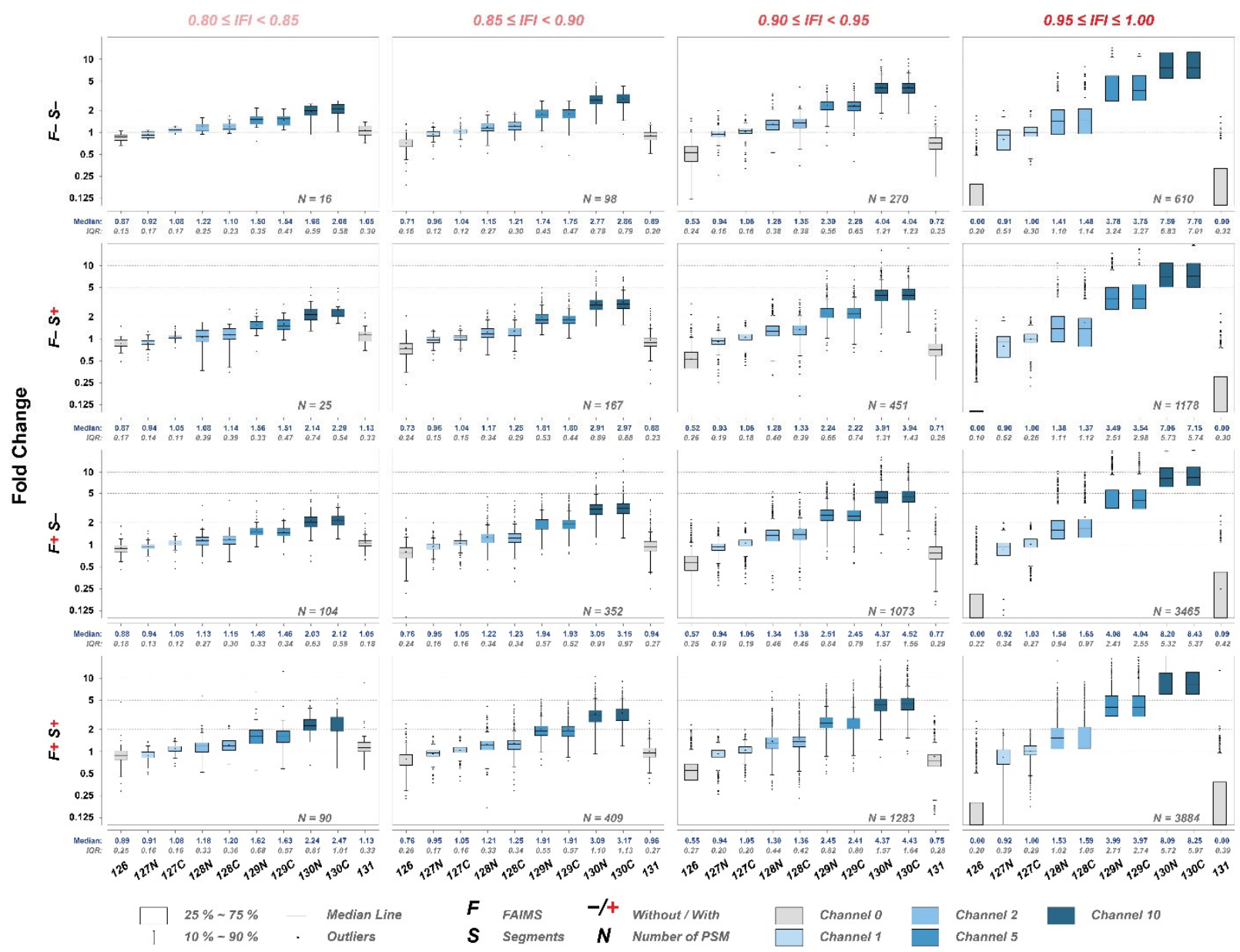

Supplementary Figure 7: Boxplot distribution of fold changes for peptides grouped by IFI. Numbers (N) of peptides per groups are indicated. 
Supplementary Table 1: Scanning ranges used for full MS scan and SIFT with and without FAIMS

\begin{tabular}{cccc}
\multicolumn{4}{c}{ FAIMS - } \\
\hline Experiment & Injection - & $\begin{array}{l}\text { CV } \\
\text { (V) }\end{array}$ & m/z range \\
\hline Rep 1 & 1 & - & $350-1150$ \\
Rep 2 & 2 & - & $350-1150$ \\
Rep 3 & 3 & - & $350-1150$ \\
Rep 4 & 4 & - & $350-1150$ \\
Rep 5 & 5 & - & $350-1150$ \\
Rep 6 & 6 & - & $350-1150$ \\
\hline
\end{tabular}

\begin{tabular}{cccc}
\multicolumn{4}{c}{ FAIMS + SIFT - } \\
\hline Experiment & Injection & $\begin{array}{l}\text { CV } \\
(\mathrm{V})\end{array}$ & $\mathbf{m} / \mathbf{z}$ range \\
\hline CV1 & 1 & -23 & $350-1150$ \\
CV2 & 2 & -30 & $350-1150$ \\
CV3 & 3 & -37 & $350-1150$ \\
CV4 & 4 & -44 & $350-1150$ \\
CV5 & 5 & -51 & $350-1150$ \\
CV6 & 6 & -58 & $350-1150$ \\
\hline
\end{tabular}

\begin{tabular}{|c|c|c|c|}
\hline Experiment & Injection & $\begin{array}{l}\text { CV } \\
\text { (V) }\end{array}$ & $\mathbf{m} / \mathbf{z}$ range \\
\hline \multirow[t]{2}{*}{ Rep 1} & 1 & - & $350-450,448-550,948-1150$ \\
\hline & $\begin{array}{l}2 \\
3\end{array}$ & - & $\begin{array}{l}548-650,848-950 \\
648-750,748-850\end{array}$ \\
\hline Rep 2 & $\begin{array}{l}4 \\
5 \\
6\end{array}$ & - & $\begin{array}{c}350-450,448-550,948-1150 \\
548-650,848-950 \\
648-750-748-850\end{array}$ \\
\hline \multirow[t]{2}{*}{ Rep 3} & $\begin{array}{l}7 \\
8\end{array}$ & - & $\begin{array}{c}350-450,448-550,948-1150 \\
548-650,848-950\end{array}$ \\
\hline & 9 & - & $648-750,748-850$ \\
\hline \multirow[t]{2}{*}{ Rep 4} & $\begin{array}{l}10 \\
11\end{array}$ & - & $\begin{array}{c}350-450,448-550,948-1150 \\
548-650,848-950\end{array}$ \\
\hline & 12 & - & $648-750,748-850$ \\
\hline \multirow[t]{2}{*}{ Rep 5} & 13 & - & $350-450,448-550,948-1150$ \\
\hline & 14 & - & $548-650,848-950$ \\
\hline Rep 6 & $\begin{array}{l}15 \\
16 \\
17 \\
18\end{array}$ & $\begin{array}{l}- \\
- \\
- \\
\end{array}$ & $\begin{array}{c}648-750,748-850 \\
350-450,448-550,948-1150 \\
548-650,848-950 \\
648-750,748-850 \\
\end{array}$ \\
\hline
\end{tabular}

\begin{tabular}{lccc} 
& \multicolumn{3}{c}{ FAIMS + SIFT +} \\
\hline Experiment & Injection & $\begin{array}{c}\text { CV } \\
\text { (V) }\end{array}$ & m/z range \\
\hline CV1 Seg1 & 1 & -23 & $350-450,548-650,648-750$, \\
CV1 Seg2 & 2 & -23 & $948-1150$ \\
CV2 Seg1 & 3 & -30 & $350-450,548-650,748-850$ \\
CV2 Seg2 & 4 & -30 & $448-550,648-750$ \\
CV2 Seg3 & 5 & -30 & $848-950,948-1150$ \\
CV3 Seg1 & 6 & -37 & $350-450,548-650$ \\
CV3 Seg2 & 7 & -37 & $448-550,748-850$ \\
CV3 Seg3 & 8 & -37 & $648-750,848-950,948-1150$ \\
CV4 Seg1 & 9 & -44 & $350-450,448-550,848-950$, \\
CV4 Seg2 & 10 & -44 & $548-650,648-750,748-850$ \\
CV5 Seg1 & 11 & -51 & $350-450,448-550,548-650$ \\
CV5 Seg2 & 12 & -51 & $648-750,748-850,848-950$, \\
CV6 Seg1 & 13 & -58 & $350-450,448-550,548-650$ \\
CV6 Seg2 & 14 & -58 & $648-750,748-850,848-950$, \\
& & & $948-1150$ \\
\hline
\end{tabular}

